\title{
OBJETO DE APRENDIZAGEM E ESPORTE ADAPTADO: APONTAMENTOS A PARTIR DE UM ESTUDO DE CASO
}

\author{
Andiara Dewes, UFSM, andiaraeduc@gmail.com \\ Roberta Feck, UFSM, roberta.feck@ @otmail.com \\ Ana Cláudia Oliveira Pavão, UFSM, anaclaudiaoliveira.pavao@gmail.com
}

Resumo: No contexto contemporâneo, permeado por exigências e desafios diversos em todos os âmbitos, a utilização das tecnologias tem proporcionado muitas possibilidades. Dentre estas, destaca-se a produção de objetos de aprendizagem (OA), os quais têm contribuído com os processos de ensino e de aprendizagem, tanto em espaços formais de escolarização como em ambientes que não sejam uma sala de aula. Nessa perspectiva, voltou-se o olhar para os OA no contex to do esporte adaptado. Desse modo, a partir do desenvolvimento, aplicação e avaliação de um OA, tem-se como objetivo identificar se um OA tem potencialidades para qualificar os processos de ensino e de aprendizagem de Basquetebol em Cadeira de Rodas-BCR, envolvendo uma movimentação de ataque. Trata-se de uma pesquisa qualitativa do tipo estudo de caso, tendo como participantes integrantes de uma equipe praticante dessa modalidade. Evidenciou-se que os OA contribuem nos processos de ensino e de aprendizagem do BCR, pois se tornam qualificadores e potencializadores de aprendizagem. Ao possibilitar analisar o jogo de uma nova perspectiva, os sujeitos qualificam o processo de entendimento da jogada e, assim, pode resultar em uma potencialização ao permitir que o jogador analise outras possibilidades de movimentações. Destaca-se que o OA teve uma avaliação satisfatória na dimensão técnica do objeto.

Palavras-chave: objeto de aprendizagem; esporte adaptado; ensino e aprendizagem.

\section{LEARNING OBJECT AND ADAPTED SPORT: APPOINTMENTS FROM A CASE STUDY}

Abstract: In the contemporary context, permeated by diverse demands and challenges in all areas, the use of technologies has provided many possibilities. Among these, we highlight the production of learning objects (OA), which have contributed to the teaching and learning processes, both in formal school spaces and in non-classroom settings. From this perspective, the curiosity to look for OA in the context of adapted sport arose. Thus, from the development, application and evaluation of an OA, the objective is to identify if an OA contributes to the teaching and learning process of Wheelchair Basketball (BCR). It is a qualitative research of the type of case study, having as participants members of a team practicing the modality. It has been shown that the OA contribute to the teaching and learning processes of the BCR, as they become qualifiers and enhancers. By making it possible to analyze the game from a new perspective, they qualify the process of understanding the play and thus can result in a potentialization by allowing the player to analyze other possibilities of moves. It is noteworthy that OA had a satisfactory evaluation in the technical dimension of the object.

Keywords: learning object; adapted sport; teaching and learning. 


\section{Apontamentos Introdutórios}

Os tempos e espaços que proporcionam possibilidades de construção de aprendizagens, cada vez mais são permeados por aspectos e elementos oriundos da evolução tecnológica das últimas décadas. Novas oportunidades emergem desse contexto, bem como novas exigências, desafios e possibilidades. Compreender o aparato tecnológico que se dispõem atualmente como meios para a qualificação e potencialização dos processos de ensino e de aprendizagem, implica em entender como esses dispositivos/mecanismos/estratégias permeiam e constituem o cotidiano, o ser e o estar no século XXI.

Nessa perspectiva, os Objetos de Aprendizagem (OA) se apresentam como um novo leque de possibilidades para qualificar e potencializar os processos de ensino e de aprendizagem. De forma conceitual, e de acordo Tarouco, Fabre e Tamusiunas (2003) objetos de aprendizagem são quaisquer recursos, que apoiem o processo de aprendizagem, e que possam ser reutilizáveis, visando potencializar as situações de aprendizagem nos locais que possam ser utilizados. Também, encontramos autores como Wiley (2000), um dos principais pesquisadores sobre objetos de aprendizagem, que considera OA "qualquer recurso digital que possa ser reutilizado para suporte ao ensino" (p. 03).

Com isso, a partir de uma disciplina de graduação de uma instituição federal de ensino superior, do curso de educação especial, licenciatura noturno, envolvendo as Tecnologias da Informação e da Comunicação (TIC), surgiu a possibilidade e o interesse de realização deste estudo. Com isso, neste artigo são apresentadas discussões decorrentes de um projeto de desenvolvimento e aplicação de um OA no esporte adaptado, especificamente, no Basquetebol de Cadeira de Rodas (BCR).

Mozzaquatro e Otto (2010) afirmam que a educação é um espaço que está passando por grandes avanços, tendo os OA como uma das ferramentas que tem contribuído para o aprendizado dos estudantes. Porém, as mesmas autoras colocam que apesar deste cenário de crescimento na área educacional, ainda, há pessoas que apresentam algum tipo de deficiência que não são atingidas da forma ideal pelo uso de OA, mas há esforços para estendê-los à todas as pessoas.

Nesta direção, neste artigo tem-se como objetivo identificar se um OA tem potencialidades para qualificar os processos de ensino e de aprendizagem de BCR, envolvendo uma movimentação de ataque.

Consolida-se a relevância social e acadêmica deste estudo, pois possibilitou encontrar indicadores de qualificação do OA desenvolvido, assim como o processo de ensino e de aprendizagem do BCR. Cabe salientar que, considerando a especificidade do público para o qual o OA foi pensado, o desenvolvimento e a aplicação deste OA permitiram discutir aspectos acerca dos tempos e espaços institucionais em que esse público tem acesso ao esporte adaptado e a maneira como os OA podem contribuir tanto no que tange aos processos de inclusão, como de ensino e de aprendizagem.

Este artigo está estruturado em quatro capítulos, a partir desta Introdução. O referencial teórico apresenta as teorias de base que versam acerca do conceito e caracterização de Objeto de Aprendizagem e de Esporte Adaptado (EA), especificamente Basquete em Cadeira de Rodas. O terceiro capítulo apresenta o delineamento metodológico caracterizado por uma pesquisa qualitativa, descritiva-exploratória do tipo estudo de caso. O quarto capítulo analisa os resultados encontrados à luz das teorias que dão suporte ao estudo. No quinto e último capítulo estão os apontamentos conclusivos da pesquisa, seguido das referências utilizadas. 


\section{Referencial Teórico}

\subsection{Objeto de Aprendizagem}

O advento das TIC impulsionadas pela disseminação do acesso à rede mundial de computadores, ainda no século passado provocaram iniciativas que resultaram em novas tendências de utilização dos novos recursos e mecanismos disponíveis e prontas a serem exploradas. Para Tarouco et. al (2006), "a tecnologia é um agente de mudança que com suas inovações tecnológicas têm transformado algumas realidades educacionais, provocando mudança de paradigmas no modo como as pessoas aprendem e como são ensinadas" (p. 02).

A facilidade na criação e disseminação de produtos e ideias por meio das TIC possibilitaram que novas formas de usufruir desses aparatos fossem construídas, bem como incorporadas ao cotidiano da sociedade. Nessa direção, conforme aponta Sabbatini (2012), emergem no cenário contemporâneo os OA, principalmente, na última década.

Neste sentido, Aguiar e Flôres (2014) afirmam que o OA é uma ferramenta que oferece vantagem para a aprendizagem e instrução podendo ser utilizado para o ensino de diferentes conteúdos. Salientam ainda que, a flexibilidade e a possibilidade de reutilização são algumas de suas características facilitando a disseminação do conhecimento.

Nessa perspectiva, Sabbatini (2012) indica que OA distinguem-se por meio de suas características de outros recursos didáticos, salientando características como a reutilização em outros contextos; portabilidade por meio, de diferentes plataformas; modularidade podendo conter ou estar em outros objetos; autossuficiência, não dependendo de outros objetos e descritos por metadados, como por exemplo, autor, palavra-chave, dentre outros.

Assim, compreende-se que os OA tornam-se novas possibilidades de, para além de apenas integrar as TIC e as produções decorrentes do uso delas, qualificar e potencializar os processos de ensino e, consequentemente, de aprendizagem. Também, configuram-se, em meios para contribuir em processos de inclusão, pois permitem apresentar ao sujeito novas perspectivas de conhecer, interagir e/ou mesmo compreender determinada informação, conhecimento, instrução, orientação.

\subsection{Esporte Adaptado (EA) - Basquete em Cadeira de Rodas}

O EA surgiu, principalmente, pós II Guerra Mundial, em um contexto de reabilitação de soldados que retornavam principalmente com lesões medulares e amputações. Buscou-se na atividade física a possibilidade de integração e interação evidenciando as potencialidades das pessoas com deficiência (ARAÚJO, 1998).

Atualmente, o esporte, muito além do processo de reabilitação, tem também como finalidade no âmbito da educação, do lazer e do rendimento, assim Silva (1999) afirma que, por meio de uma evolução e enriquecimento de seus conceitos iniciais, o esporte vem possibilitar além da superação física e psicológica, a inclusão ao meio social, um reconhecimento das habilidades, capacidades e potencialidades envolvidas no esporte da pessoa com deficiência.

De maneira conceitual, segundo Araújo (1998), o esporte adaptado é uma modalidade convencional que sofre algumas alterações para que possa ser acessível a todos, ou a um grupo com limitações específicas, ou ainda esportes novos, criados para um determinado grupo que apresenta uma deficiência. 
No Brasil, o esporte adaptado surgiu em 1958, onde brasileiros paraplégicos e tetraplégicos voltavam de seus respectivos tratamentos no exterior e resolveram compartilhar suas experiências e proporcionar a prática desportiva para as pessoas com deficiência que aqui residiam.

Com o apoio da ANDE - Associação Nacional Desporto para Excepcional, surgida em 1975, essa prática esportiva tinha por objetivo prevenir e tratar lesões ou doenças. Devido às diversas limitações e o grande interesse das pessoas com necessidades especiais, foi necessário criar novas entidades para atender todas as pessoas que se interessavam pela prática esportiva.

Em 1957, se formava a primeira equipe de BCR no Brasil e em fevereiro de 1958, iniciaram-se os treinamentos da modalidade, no Hospital das Clínicas em São Paulo (ARAÚJO, 1998).

O esporte BCR é dirigido às pessoas com deficiência nos membros inferiores, tais como lesão medular, sequelas de poliomielite, má formação congênita, amputações (GORLA, 2010). As regras são basicamente as mesmas do Basquetebol convencional, com algumas modificações ou acréscimo em comparação com as regras da Federação Internacional de Stoke Mondeville e o sistema de classificação funcional para assegurar a competição justa entre os atletas/praticantes e oportunizar a participação de pessoas com diferentes níveis de lesões. Cada equipe é composta de 5 jogadores e poderá somar no máximo 14 (quatorze) pontos, sabendo-se que atualmente são 5 (cinco) classes funcionais, variando de 1,0 (um) ponto até 4,5 (quatro e meio) pontos. (IWBF, 2002)

\section{Percurso Metodológico}

Esse estudo se caracteriza como uma pesquisa do tipo qualitativa (THOMAS, NELSON e SILVERMAN, 2012). Quanto aos objetivos, optou-se por uma pesquisa descritiva-exploratória, que para Gil (2008) descreve as características de determinadas populações ou fenômenos, bem como tem como objetivo proporcionar maior familiaridade com o problema (explicitá-los). Em relação aos procedimentos, refere-se a um estudo de caso.

Ao referenciar Yin (2010), explica que “(...) o estudo de caso é uma investigação empírica que investiga um fenômeno contemporâneo em profundidade e em seu contexto de vida real" (p. 39). Fazer uso de um estudo de caso visa explorar situações da vida real, descrever a situação do contexto em que está sendo feita determinada investigação e explicar as variáveis causais de determinado fenômeno em situações que não se podem utilizar experimentos.

A escolha do público é do tipo intencional que "consiste em selecionar um subgrupo da população que, com base nas informações disponíveis, possa ser considerado representativo de toda a população" (GIL, 2008, p. 94). Neste sentido, fizeram parte do estudo cinco (5) pessoas com deficiência física, participantes de uma equipe de Basquetebol em Cadeira de Rodas, sendo que todos estão vinculados a um projeto extensionista de uma universidade federal do sul do país. Acerca dos participantes, todos eram do sexo masculino, com faixa etária de 30 a 40 anos, praticantes da modalidade há mais de dois anos.

Como fases metodológicas destacamos que a pesquisa envolveu inicialmente o desenvolvimento do OA, realizado com as ferramentas disponíveis em um aplicativo de apresentação de slides, com a inserção de vídeos de apoio para visualização da movimentação do ataque do BCR. Para os vídeos, foi utilizado um aplicativo gratuito 
denominado TacticalPad Basquete $^{1}$, no qual há uma prancheta virtual que disponibiliza o desenho completo de uma quadra de basquete, permitindo criar ícones e desenhos desde formações táticas até a escalação da equipe. Também como forma de acessibilidade, o objeto contou com áudios reforçando as instruções apresentadas em cada tela. A Figura 1 apresenta duas interfaces do OA.

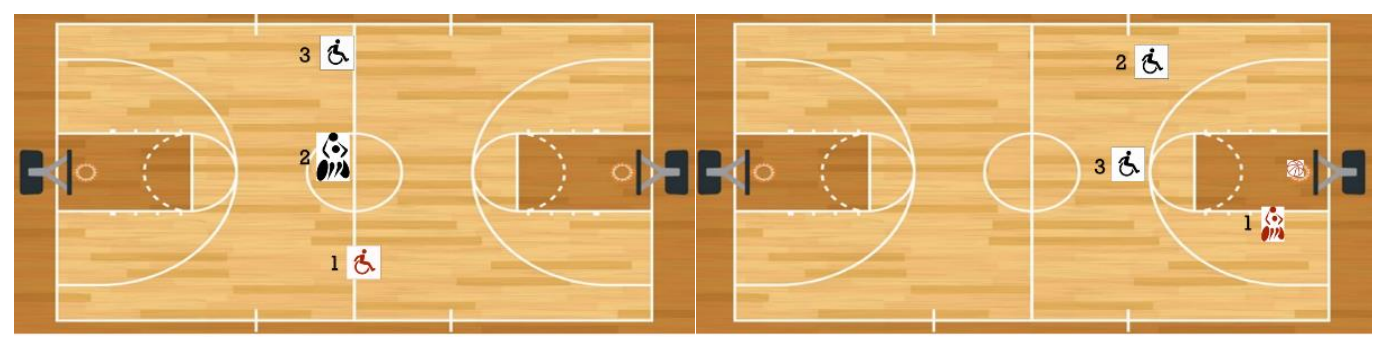

Figura 1- OA Basquetebol de Cadeira de Rodas

$\mathrm{Na}$ fase seguinte foi realizada a apresentação do objeto de forma individual, sendo então utilizado pelos participantes da pesquisa. Por fim, foi aplicado o questionário de avaliação.

Desta forma, para a coleta de dados foi realizado o contato com a coordenadora do projeto de extensão e após, com cada participante, verificando o interesse para a participação da pesquisa. Foram apresentados os objetivos do estudo, sendo agendado os dias para a apresentação e utilização do OA e para aplicação do questionário de avaliação com os participantes. Para avaliar o OA foi utilizado um questionário fechado com respostas fechadas/dirigidas organizadas em duas dimensões de avaliação: técnica e pedagógica. As respostas a cada aspecto avaliado estão categorizadas de forma quantitativa, sendo 1 quando muito insatisfeito, 2 insatisfeito, 3 considerado nem bom e nem ruim, 4 satisfeito e 5 muito satisfeito.

Compreendemos as dimensões de avaliação escolhidas a partir da definição de Melo (2009). Para a autora

\begin{abstract}
a dimensão pedagógica é demarcada pelas preocupações com a aprendizagem, definição de conceitos e de teorias acerca do conhecimento. Enquanto a dimensão ergonômica importar--se--á com a sua utilidade, adequação aos objetivos pretendidos, navegabilidade e facilidade de uso. E a dimensão de caracterização dos objetos corresponde ao atendimento das características básicas para que o software seja considerado como um objeto de aprendizagem. (p. 37).
\end{abstract}

Tanto a apresentação e utilização do objeto, quanto a aplicação do questionário foram realizados de maneira individual com cada participante pelas pesquisadoras responsáveis. Destaca-se que todos os participantes estavam cientes dos objetivos e procedimentos do presente estudo e assinaram um Termo de Consentimento Livre e Esclarecido, aceitando participar do estudo.

A análise dos dados obtidos da escala Likert foi realizada com estatística descritiva, tendo como base as respostas mais frequentes.

\title{
4. Resultados E Discussões
}

Por meio da realização da avaliação pelos participantes estabelece-se a possibilidade de, além de conhecermos a percepção acerca da utilização do OA no esporte

\footnotetext{
${ }^{1}$ Disponível em: < http://www.baixaki.com.br/download/tacticalpad-basquete.htm >. 
adaptado, revisarmos aspectos que precisam ser melhorados, visando sua própria qualificação. Neste sentido, destacamos que o foco da avaliação deste objeto está centrada no quanto ele pode contribuir na relação de ensino e a aprendizagem ao participante, envolvendo uma movimentação de ataque do BCR. Conforme estudo de Flôres e Tarouco (2008), ao se construir um OA deve se pensar na interação que o objeto proporciona, tendo como objetivo maior uma aprendizagem significativa, sendo que esse recurso venha ser um facilitador neste processo.

Os Quadros 1 e 2, a seguir, referem-se respectivamente às dimensões técnica e pedagógica analisadas no OA. Os quadros utilizam da Escala Likert para avaliação, nos quais o numeral 1 indica Muito Insatisfeito, 2- Insatisfeito, 3- Nem bom, nem ruim, 4Satisfeito e 5- Muito Satisfeito.

Desta forma, quanto a dimensão técnica tem-se o seguinte panorama acerca do objeto aplicado, conforme demonstrado no Quadro 1.

\begin{tabular}{|c|c|c|c|c|c|c|}
\hline Dimensão de & Aspecto avaliado & \multicolumn{5}{|c|}{ Avaliação } \\
\hline \multirow{6}{*}{$\begin{array}{l}\text { Dimensão } \\
\text { Técnica do } \\
\text { OA }\end{array}$} & & $\begin{array}{c}1 \\
\text { Muito } \\
\text { Insatisfeito }\end{array}$ & $\begin{array}{c}2 \\
\text { Insatisfeito }\end{array}$ & $\begin{array}{c}3 \\
\text { Nem bom, } \\
\text { nem ruim }\end{array}$ & $\begin{array}{c}4 \\
\text { Satisfeito }\end{array}$ & $\begin{array}{c}5 \\
\text { Muito } \\
\text { Satisfeito } \\
\end{array}$ \\
\hline & $\begin{array}{l}\text { A linguagem utilizada é } \\
\text { adequada? }\end{array}$ & & & & P2; P3 & P1; P4; P5 \\
\hline & $\begin{array}{l}\text { As imagens utilizadas } \\
\text { são claras? }\end{array}$ & & & & P1; P2 & P3; P4; P5 \\
\hline & $\begin{array}{l}\text { O AO se mostrou } \\
\text { adequado ao público } \\
\text { alvo apontado? }\end{array}$ & & & & $\begin{array}{l}\text { P2; P3; } \\
\text { P4 }\end{array}$ & P1; P5 \\
\hline & Feedback & & & & P4; P5 & P1; P2; P3 \\
\hline & $\begin{array}{l}\text { Adequação e coerência } \\
\text { dos conteúdos ao } \\
\text { público alvo }\end{array}$ & & & P5 & $\begin{array}{l}\text { P1; P2; } \\
\text { P3; P4 }\end{array}$ & \\
\hline
\end{tabular}

Quadro 01: Dimensão técnica: síntese das respostas do questionário de avaliações do OA.

Fonte: as autoras.

Em relação à avaliação da dimensão técnica, evidenciamos que o OA é satisfatório com tendência a muito satisfatório. Destaca-se que o conceito "satisfeito" foi indicado 13 vezes e o conceito "muito satisfeito" teve 11 indicações, distribuídos em cinco aspectos avaliados. O conceito "nem bom, nem ruim" teve 1 indicação no aspecto relativo à 'adequação e coerência dos conteúdos ao público alvo', porém quatro pontuaram estar satisfeitos. Esta avaliação indica a necessidade de revisão desse aspecto visando a qualificação, além de apontar diferentes níveis de habilidade técnica e tática dentro da mesma equipe, sendo que para alguns participantes a movimentação de BCR pode ser complexa, ao mesmo tempo que para outros, a mesma torna-se simples.

Com relação a linguagem utilizada no OA, a clareza das imagens e feedback, três dos participantes avaliaram como muito satisfeito, e dois como satisfeito. Relacionado a adequação ao público alvo apontado, três satisfeito e dois muito satisfeito. Nessa direção, entendemos que quanto à dimensão técnica atingimos o objetivo de desenvolver um OA adequado ao esporte adaptado, no caso o BCR.

Já, direcionando-se à dimensão pedagógica, de acordo com o Quadro 2, temos os seguintes resultados,

\begin{tabular}{|c|c|c|}
\hline $\begin{array}{c}\text { Dimensão de } \\
\text { avaliação }\end{array}$ & Aspecto avaliado & Avaliação \\
\hline
\end{tabular}




\begin{tabular}{|c|c|c|c|c|c|c|}
\hline \multirow{8}{*}{$\begin{array}{l}\text { Dimensão } \\
\text { Pedagógica } \\
\text { do OA }\end{array}$} & & $\begin{array}{c}1 \\
\text { Muito } \\
\text { Insatisfeito }\end{array}$ & $\begin{array}{c}2 \\
\text { Insatisfeito }\end{array}$ & $\begin{array}{c}3 \\
\text { Nem bom, } \\
\text { nem ruim }\end{array}$ & $\begin{array}{c}4 \\
\text { Satisfeito }\end{array}$ & $\begin{array}{c}5 \\
\text { Muito } \\
\text { Satisfeito }\end{array}$ \\
\hline & $\begin{array}{l}\text { A linguagem utilizada } \\
\text { no OA favorece o } \\
\text { entendimento e a } \\
\text { aprendizagem da jogada }\end{array}$ & & & & P4; P5 & P1; P2; P3 \\
\hline & $\begin{array}{l}\text { O OA permite que os } \\
\text { alunos realizem as } \\
\text { atividades sem } \\
\text { intervenção do } \\
\text { professor }\end{array}$ & & P5 & P2 & P3; P4 & P1 \\
\hline & $\begin{array}{l}\text { Os conteúdos são } \\
\text { abordados de maneira } \\
\text { clara e precisa }\end{array}$ & & & & P5 & $\begin{array}{l}\text { P1; P2; P3; } \\
\text { P4 }\end{array}$ \\
\hline & $\begin{array}{l}\text { Possibilidade de reuso } \\
\text { em outros contextos } \\
\text { pedagógicos }\end{array}$ & & & $\mathrm{P} 2$ & $\begin{array}{l}\text { P3; P4; } \\
\text { P5 }\end{array}$ & P1 \\
\hline & $\begin{array}{l}\text { Qualidade dos conceitos } \\
\text { abordados }\end{array}$ & & & & $\begin{array}{l}\text { P2; P4; } \\
\text { P5 }\end{array}$ & P1; P3 \\
\hline & $\begin{array}{l}\text { Adequação dos } \\
\text { conteúdos }\end{array}$ & & & & $\begin{array}{l}\text { P2; P3; } \\
\text { P4; P5 }\end{array}$ & P1 \\
\hline & $\begin{array}{l}\text { Adequação dos } \\
\text { objetivos }\end{array}$ & & & & $\begin{array}{l}\text { P2; P3; } \\
\text { P4; P5 }\end{array}$ & P1 \\
\hline \multicolumn{2}{|c|}{$\begin{array}{l}\text { O que considera relevante e não foi } \\
\text { contemplado nas perguntas? } \\
\text { Sugestões: }\end{array}$} & \multicolumn{5}{|c|}{$\begin{array}{l}\text { Pode melhorar para situação real de jogos, ou seja, com } 5 \text { jogadores, pois } \\
\text { envolve somente } 3 \text {. (P1) } \\
\text { Depende de quem ver, não vai ter total compreensão. Exemplo do } \\
\text { companheiro da equipe. (P5) }\end{array}$} \\
\hline
\end{tabular}

Quadro 02: Dimensão pedagógica: síntese das respostas do questionário de avaliações do OA. Fonte: as autoras.

O Quadro 2 apresenta os resultados, no que tange à avaliação da dimensão pedagógica. Assim, pode-se considerar que o OA é satisfatório, pois como o conceito "satisfeito" foi indicado 19 vezes e o conceito "muito satisfeito" teve 13 indicações, distribuídos em sete aspectos avaliados.

Destaca-se que o conceito "nem bom, nem ruim" teve 2 indicações. O aspecto relativo à "o OA permite que os alunos realizem as atividades sem intervenção do professor?" teve uma indicação como "nem bom, nem ruim" e uma indicação de "insatisfeito". Essa avaliação indica que o professor se torna ponto fundamental no processo de ensino e aprendizagem também na área da educação física envolvendo a prática esportiva, sendo o objeto um recurso de apoio que facilita e potencializa a aprendizagem, mas que não substitui o professor. Conforme Geller (2004), o computador torna-se um grande aliado do professor, sendo este um recurso mediador de uma aprendizagem, mas sua utilização não substitui o professor.

O aspecto "possibilidade de reuso em outros contextos pedagógicos" teve uma indicação de "nem bom, nem ruim". Neste sentido, justificamos a falta da inclusão e expansão da prática de esportes adaptados em diferentes contextos, como por exemplo, na escola, sendo esta uma realidade que influencia nas respostas dada pelos participantes, visto a compreensão desta realidade.

Quando perguntados se os conteúdos são abordados de maneira clara e precisa, quatro responderam estar muito satisfeito e um satisfeito. Quanto à qualidade dos conceitos abordados pelo OA, dois avaliaram como muito satisfeitos e três como satisfeito. Por fim, quanto à adequação do conteúdo e dos objetivos, quatro participantes avaliaram como satisfeito e um como muito satisfeito.

Destaca-se que houve a utilização do espaço "observação" por dois dos participantes da pesquisa. Um destacou que o objeto envolveu apenas três jogadores, sendo que poderia ser desenvolvido de modo a abranger os cinco jogadores em quadra, abordando uma situação real de jogo. Já o outro indicou que evidenciou na utilização do OA, que dependendo de quem for utilizar, não vai ter total compreensão, exemplificando 
o companheiro de equipe, o qual precisa de reforços para boa compreensão, quando envolve situações de jogo.

Por fim, pode-se concluir que os resultados encontrados apontam que o OA desenvolvido atua como um facilitador no processo de ensino e aprendizagem do BCR, contribuindo para o entendimento da movimentação de ataque.

\section{Apontamentos Conclusivos}

O desenvolvimento e a aplicação de um OA voltado ao BCR possibilitou evidenciarmos contribuições no processo de ensino e de aprendizagem desta modalidade, quando da sua utilização. Desta forma, infere-se a necessidade e importância de ampliar o uso de OA ao público com deficiência, percebendo as contribuições neste estudo, considerando que, ainda pessoas com algum tipo de deficiência não são favorecidas e atingidas pelo uso deste recurso. Isso poderá ser modificado, por meio da capacitação de professores quanto ao uso de OA para potencializar o ensino e aprendizagem, nos mais variados conteúdos e para públicos diversos.

Quanto ao objeto desenvolvido, destaca-se como resultado uma avaliação satisfatória na dimensão técnica do objeto. Evidenciou-se que o OA contribui nos processos de ensino e de aprendizagem de uma movimentação de ataque do BCR, pois se torna qualificador e potencializador desse processo. Ao analisar o jogo de uma nova perspectiva, o OA qualifica o entendimento da jogada e, assim, pode resultar em uma nova aprendizagem, ao permitir que o jogador analise outras perspectivas de movimentações. Essas analises não omitem apontar a limitação técnica que o OA apresenta, pelo fato de ter sido desenvolvido com um aplicativo de apresentação de slides e, por isso com interações limitadas. No entanto, mesmo com baixo índice de interações, mostrou-se com possibilidades reais de aprendizagem dos usuários.

A partir das avaliações realizadas pelos jogadores identificou-se que um OA, quando tem como temática uma situação real que é vivenciada por quem irá utilizar o objeto, precisa abarcar a similaridade do cenário/contexto no OA com o real. Evidenciouse, assim, que quanto mais próximo ao real, mais qualificado se torna o OA. Ressalta-se também a importância de adequar os conteúdos ao nível de desenvolvimento dos usuários do OA, sendo o professor fundamental neste processo.

Portanto, por meio dos apontamentos realizados a partir desse estudo de caso compreendeu-se que um OA tem potencialidades para qualificar os processos de ensino e de aprendizagem também em situações que não sejam no ambiente formal de escolarização.

\section{Referências}

AGUIAR, E. V. B.; FLÔRES, M. L. P.. Objetos de aprendizagem: conceitos básicos. In.: TAROUCO, Liane M. R.; ÁVILA, B. G.; SANTOS, E. F.; BEZ, M. R.; COSTA, V. (Org.). Objetos de Aprendizagem: teoria e prática. Porto Alegre, RS: Evangraf, 2014.

\section{ARAÚJO, P. F. Desporto adaptado no Brasil: origem, institucionalização e atualidade. Brasília: INDESP, 1998.}

FLORES, M.L.P.; TAROUCO, L. M. R Diferentes tipos de objetos para dar suporte a aprendizagem. RENOTE: Revista Novas Tecnologias da Educação, Porto Alegre, V. $6 \mathrm{~N}^{\circ}$ 1, Julho, 2008. Disponivel em: < http://seer.ufrgs.br/renote/article/viewFile/14513/8438 $>$. Acesso em out de 2017. 
Geller, M. (2004), Educação a Distância e Estilos Cognitivos: construindo um novo olhar sobre os ambientes virtuais. Porto Alegre: UFRGS. Tese (Doutorado em Informática na Educação), Programa de Pós-Graduação em Informática na Educação, Universidade Federal do Rio Grande do Sul.

GIL, A. C. Métodos e técnica de pesquisa social. São Paulo, SP: Atlas, 2008.

GORLA, J. I. Handebol em Cadeira de Rodas: regras e treinamento. São Paulo, SP: Phorte, 2010.

INTERNATIONAL WHELLCHAIR BASKETBALL FEDERATION - IWBF. Internal Regulations Techinical Comission. August, 2002.

MELO, J. A. P. Avaliação de Objetos de Aprendizagem: cruzando caminhos e produzindo novos olhares. Dissertação de Mestrado. Universidade Federal de Uberlândia. Programa de Pós-graduação em Educação. Mestrado. 2009. 139 f. Disponível em: <https://repositorio.ufu.br/bitstream/123456789/13792/1/Janete.pdf>. Acesso em set de 2017.

MELO, J. A. P. Avaliação de objetos de aprendizagem: cruzando caminhos e produzindo novos olhares. Revista Latinoamericana de Tecnología Educativa - RELATEC, 8 (2), 59-75. 2009. Disponível em: <http://campusvirtual.unex.es/cala/editio/>. Acesso em out de 2017.

OTTO, C. S.; MOZZAQUATRO, P. M.. Desenvolvimento de um Objetos e Aprendizagem para portadores de visão subnormal a partir do uso de conceitos e técnicas de acessibilidade. São Paulo, SP: Abril, 2010.

SABBATINI, M. Reflexões Críticas Sobre o Conceito de Objeto de Aprendizagem Aplicado ao Ensino de Ciências e Matemática. EM TEIA - Revista de Educação Matemática e Tecnológica Iberoamericana. vol. 3. n. 3. Recife, PE: 2012. Disponível em: http://www.pucrs.br/famat/viali/tic_literatura/artigos/objetos/Sabatini_Marcelo.pdf.

Acesso em set de 2017.

SILVA, A. C. Atleta portador de deficiência. In: GHORAYEB, N.; BARROS, T. O exercício: Preparação fisiológica, avaliação médica - Aspectos especiais e preventivos. São Paulo, SP: Atheneu, 1999.

TAROUCO, L. M. R.; KONRATH, M. L. P.; CARVAlHO, M. J. S.; ÁVILA, B. G. Formação de professores para produção e uso de objetos de aprendizagem. RENOTE Revista Novas Tecnologias na Educação. Porto Alegre, RS: Centro Interdisciplinar de Novas Tecnologias na Educação (UFRGS), v. 4, n. 1, julho de 2006. Disponível em:http://penta2.ufrgs.br/edutools/tutcmaps/tutindicecmap.htm. Acesso em out de 2017.

TAROUCO, L. M. R.; FABRE, M.-C. J. M.; TAMUSIUNAS, F. R.. Reusabilidade de objetos educacionais. RENOTE: Revista Novas Tecnologias da Educação, Porto Alegre, v. 1 n. $1, \quad$ p. 1-11, fev. 2003. Disponível em: $\langle$ http://www.nuted.ufrgs.br/oficinas/criacao/marie_reusabilidade.pdf $>$. Acesso em out de 2017. 
THOMAS, J. R.; NELSON, J.K.; SILVERMAN, S.J. Métodos de pesquisa em atividade física. $6^{\text {a }}$ ed. Porto Alegre, RS: Artmed, 2012.

WILEY, D. A. Conecting learning objects to instructional theory: a definition, a methaphor anda a taxonomy. The Instructional Use of Learning Objets, 2001. Disponível em: <http://reusability.org./ read/chapters/wiley.doc> Acesso em set de 2017.

YIN, R. K. Estudo de caso: planejamento e métodos. 4. ed. Porto Alegre, RS: Bookman, 2010. 